\title{
ASSESSMENT OF COLLABORATIVE NETWORKS STRUCTURAL STABILITY
}

\author{
Vera Tolkacheva ${ }^{2}$, Dmitry Ivanov $^{1}$, Alexander Arkhipov ${ }^{2}$ \\ 'Chemnitz University of Technology, GERMANY \\ dmitri.ivanov@mail.ru \\ ${ }^{2}$ Saint Petersburg State University of Technology and Design, RUSSIA \\ a arkhipov@sutd.ru \\ vera_tolkacheva@mail.ru
}

\begin{abstract}
Subject of this contribution is to develop a framework for assessment of collaborative networks (CN) structural stability on the stage of the CN design. $C N$ design is a critical source of competitive advantage given that as much as $80 \%$ of total product cost mav be fixed by these decisions. The importance of the stable $C N$ structuring is evident. The elaborated framework aims to develop a technique for simultaneous CN structuring and its stability assessment based on parametric analysis of possible participants and integral stability assessment. This procedure is based on the application of special Index of Structure Consolidation (ISC), which makes it possible to estimate the project stability. The index shows mutual interest between $C N$ partners and the coordinator. This index should be taken into account while taking decision about the final structure of the network and its application claims for fuzzy logic introduction. It makes it possible to increase the quality of decision-making about the $C N$ configuration under the terms of uncertainty.
\end{abstract}

\section{INTRODUCTION}

$\mathrm{CN}$ design is a critical source of competitive advantage given that as much as $80 \%$ of total product cost may be fixed by these decisions (Harrison et al., 2005). The importance of the stable $\mathrm{CN}$ structuring is evident. One of the $\mathrm{CN}$ structuring challenges is a combined formation of the $\mathrm{CN}$ structural-functional-informational configuration and estimation of the $\mathrm{CN}$ execution stability. An important point of such simultaneous formation consists in ensuring of the business-processes continuity, information availability, and system stability. To answer this challenge, we introduce a framework for assessment of collaborative networks $(\mathrm{CN})$ structural stability on the stage of the $\mathrm{CN}$ design.

\section{LITERATURE ANALYSIS}

$\mathrm{CN}$ functioning is challenged by high uncertainty. This leads to perturbations and deviations during the $\mathrm{CN}$ execution. Stability is an appropriate category for the increasing quality of the $\mathrm{CN}$ modelling and decision making under the terms of uncertainty. In general case stability analysis consists in investigation of influence of the $\mathrm{CN}$ execution parameters deviations on the final $\mathrm{CN}$ goals. Stability analysis is especially useful in the situations, which are characterized by high level of uncertainty, which does not allow producing deterministic or stochastic models. The stability analysis allows proofing execution plan feasibility, selecting a plan with the sufficient stability degree from a set of alternative plans, determining $\mathrm{CN}$ execution bottle-necks and steps for their strengthening.

Tolkacheva, V., Ivanov, D., Arkhipov, A., 2007, in IFIP International Federation for Information Processing, Volume 243, Establishing the Foundation of Collaborative Networks; eds. Camarinha-Matos, L., Afsarmanesh, H., Novais, P., Analide, C.; (Boston: Springer), pp. 75-82. 
The concept of 'stability' plays a fundamental role in the systems theory. The sense of this term in general is equal for different types and classes of systems. It consists in limited reaction of a system (bounded output) on scale-limited (bounded input) entering impacts (controlled and non-controlled). In the systems and control theories is usually used a term BIBO (Bounded Input Bounded Output)-Stability. In mathematics, stability theory deals with the stability of the solutions of differential equations and dynamical systems (Rouche et al., 1977). Stability can be estimated by means of different approaches such as Nyquist, Hurwitz, Routhor with Crossover Modell von McRuer.

In production and logistics, the issue of plan stability has attracted increased attention and interest in recent years. This is mainly due to an increasing integration of planning systems both within and across companies in supply chain management Heisig, (2006). (Fox et al., 2006) proposes a framework for using plan stability metric in the plan adaptation issues. The authors demonstrate empirically based on the local search strategy that the proposed plan repair strategy achieves more stability than replanning without stability consideration. (Groson et al., 2005) consider the order stability issues in supply chains with the focus on coordination to avoid the bullwhip effect. These experiments follow the standard protocol of the Beer Distribution Game. Approach, described in (Kulba, 2006), introduces the term of structural-technological reserve for increasing stability of manufacturing systems and proposes a Petri-net based solution method.

A special feature of the $\mathrm{CN}$ stability analysis consists in adjustment actions elaborated by managers (not by machines) in a combination of centralized and decentralized management. The $\mathrm{CN}$ differs from a physical system. The latter is remarkable for its planning mechanisms, which have some elements of subjectivism. That is why it becomes necessary to broaden the sense of 'stability' term while CN considering. In paper (Ivanov et al., 2006), we introduced a framework of CN stability analysis and presented a conceptual model of $\mathrm{CN}$ stability analysis and its dynamical interpretation. In this paper, we put the emphasis on a specific aspect of the CN stability analysis - analysis of collaboration stability. The elaborated framework aims to develop a technique for simultaneous $\mathrm{CN}$ structuring and its stability assessment based on parametric analysis of possible participants and integral stability assessment. This procedure is based on the application of special index of structure consolidation (ISC), which makes it possible to estimate the project stability.

\section{FRAMEWORK FOR COLLABORATIVE NETWORK SYNTHESIS}

At the $\mathrm{CN}$ configuration stage the aim of a $\mathrm{CN}$ coordinator is to synthesize alternative structures of $\mathrm{CN}$ and range them due to their preference. This procedure is carried out on the base of two structured setss: the set of potential executors and the set of alternative project structures.

Project structuring. At this step the goal of coordinator consists in project description and structuring according to the proper level of decomposition. In complex projects different operations have different importance for the final result. That is why the importance level of each operation must to be taken into account. When coordinator is in charge of several projects, there may be situations, when the same competency is needed for different projects at the same time. These projects should not be considered independent, because they compete for the same resource. In order 
to analyse such "linked" projects together we propose to present them as a joined structure scheme that we call technological network. The model of technological network is an oriented graph. Its heads are considered competencies that are necessary for project realisation, and its edges serve to show the logic sequence of operations. Weights of each head reflect the volume of the competency that is needed for projects performance (e.g., the total working time to execute a concrete technological operation on a concrete machine). Finally, in the end of this step coordinator obtains properly structured projects that are considered technological network.

Executors structuring. Using common information breeding environment, coordinator analyses possible executors for his technological network. Each potential participant is characterised by several competencies as well as by additional characteristics (an ability to perform their obligations in the specified terms, a presence of free resources to accomplish the order in full, a proper quality level of delivered products, a price per unit, a level of additional costs, a risk level of order execution failure).

Executors structuring is carried out in two phases: i) analysing the whole set of information breeding environment members, coordinator selects potential participants for his project due to their competencies; ii) coordinator analysis additional characteristics of selected potential executors for the each stage and ranges them. This procedure was thoroughly described in recent works (Ivanov et al, 2006). First of all, it is necessary to consecutively allocate Pareto-optimal groups of alternative executors and give a rank to each group. Then applying the special method, proposed by authors, which is based on the concept of "curves of indifference", coordinator ranges the members of each determined group. By the end of this step coordinator has several alternative executors for each project stage that are ranged due to their preference level.

$\mathrm{CN}$ synthesis. There are different approaches to $\mathrm{CN}$ synthesis that depend on whether a technological network and a number of possible executors are fixed or not. In this paper we consider, that projects are independent (they do not compete for the same resources), the structure of technological network is fixed and coordinator is provided with all necessary information about potential members.

Coordinator should built different alternative variants of $\mathrm{CN}$ structures, assess them and select the most preferable. According to different requirements to the result, several procedures may be applied: i) full number of combinations of executors for each project stage; ii) formation of the sole $\mathrm{CN}$ structure from the preset reduced number of best executors for each separate stage (local optimization); iii) reduced number of $\mathrm{CN}$ alternative combinations.

The third procedure seems to be the most advantageous. This method provides us with common search scheme, whose limiting cases are the procedures described above - full number of combinations and local optimization. Introduction of restrictions on executor ranks is important, because it allows coordinator to reduce the space of search considerably. Synthesis of $\mathrm{CN}$ alternative variants is carried out by introduction of executors (from the obtained subset) in the next $\mathrm{CN}$ variant according to their rank. The result of this procedure is the set of $\mathrm{CN}$ alternative structures ranged due to their preference level. This is the most flexible form of result as it lets the coordinator operatively maneuver in case various sorts of failures or deviations during project performance occur. Thus, the result of the given step is the synthesis of either one $\mathrm{CN}$ structure that is most preferable for the coordinator, or the sequence of $\mathrm{CN}$ structures ordered due to their preference level. 


\section{INTEGRAL STABILITY ASSESSMENT OF THE CN STRUCTURE}

Now coordinator should carry out the analysis of the constructed CN. First of all it is necessary to evaluate the internal "affinity", "durability" of the synthesized CN. As $\mathrm{CN}$ members are independent active subjects and their aims may change under market environment, the assessment of potential reliability (structure stability) seems to be much more complicated. There may be situations, when $\mathrm{CN}$ members are willingly to go out of the project, and coordinator - to change the executor. An active behavior of $\mathrm{CN}$ participants together with their insufficient consolidation in the frameworks of $\mathrm{CN}$ can lead to project failure that, obviously, is extremely undesirable for the coordinator, responsible for its realization.

In this paper we propose an approach to the $\mathrm{CN}$ reliability assessment, which is based on the application of special index of structure consolidation (ISC). This index shows mutual interest between $\mathrm{CN}$ partners and the coordinator and makes it possible to estimate how successful the project will be. This index should be taken into account while taking decision about the final structure of the collaborative network. To determine the ISC we offer the following algorithm.

\section{The evaluation of coordinator's interest in i-executor}

Let us consider, that i-executor is described with the parameter vector $\mathbf{p}_{\mathrm{i}}=\left(\mathrm{p}_{\mathrm{i} 1}, \mathrm{p}_{\mathrm{i} 2}, \ldots, \mathrm{p}_{\mathrm{in}}\right)$. We introduce the concept of $\mathrm{i}$-executor utility and believe that it additively depends on values of parameters mentioned above:

$$
\mathbf{P}_{\mathrm{i}}=\lambda_{1} \cdot \mathrm{p}_{\mathrm{i} 1}+\lambda_{2} \cdot \mathrm{p}_{\mathrm{i} 2}+\ldots+\lambda_{\mathrm{n}} \cdot \mathrm{p}_{\mathrm{in}}
$$

where $\mathbf{P}_{\mathbf{i}}-$ i-executor utility function;

$\mathrm{p}_{\mathrm{i} 1}, \mathrm{p}_{\mathrm{i} 2}, \ldots, \mathrm{p}_{\mathrm{in}}-\mathrm{i}$-executor normalized parameters;

$\lambda_{1}, \lambda_{2}, \lambda_{n},-$ coefficients of parameter importance, determined by the coordinator.

We assume that coordinator is able to explain what values of executor's parameters he wants to see. The vector of such desirable parameters we call "ideal" vector $\mathbf{p}^{\mathbf{0}}$. So, we can determine the ideal utility function $\mathbf{P}_{\mathbf{i}}^{\mathbf{0}}$.

The index $\mathbf{U}_{\mathbf{i}}$ of coordinator's interest in i-executor we establish as follows:

$$
\mathbf{U}_{\mathbf{i}}=\mathbf{P}_{\mathbf{i}} / \mathbf{P}_{\mathbf{i}}^{\mathbf{0}} \mathbf{i}=1,2, \ldots, \mathrm{n}
$$

It is obviously that this index may alter in the interval $[0,1]$.

2. The evaluation of i-executor's interest in project by the coordinator

From the point of view of the coordinator the executor's interest in project is defined by two factors: i) the level of capacity utilization while order execution $\left(\mathrm{K}_{\mathrm{Mi}}\right)$; ii) the expected revenue $\left(\mathrm{C}_{\mathrm{i}}\right)$, i.e. the cost of the contract between the executor and the coordinator. follows:

The utility $\left(\mathbf{S}_{\mathbf{i}}\right)$ of participation in the project for the i-executor we determine as

$$
\mathbf{S}_{\mathrm{i}}=\lambda_{\mathrm{c}} \cdot \mathrm{C}_{\mathrm{i}}+\lambda_{\mathrm{k}} \cdot \mathrm{K}_{\mathrm{Mi}},
$$

where $\lambda_{c}$ and $\lambda_{k}$ - coefficients of parameter importance, determined by the coordinator.

Let us determine the vector of "ideal" values of parameters $C_{i}$ and $K_{M i}$ and, accordingly, the ideal value of utility function $\mathbf{S}_{\mathbf{i}}{ }^{\mathbf{0}}$. The latter is established for a case when executor has the maximal level of capacity utilization $\left(K_{\mathrm{Mi}}=1\right)$ and the expected revenue is "normative" for him $\left(C_{i}=C_{n o r m}\right)$.

The evaluation of i-executor's interest in project participation by the coordinator $\left(\mathbf{Z}_{\mathbf{i}}\right)$ is determined as follows: 


$$
\mathbf{Z}_{\mathbf{i}}=\mathbf{S}_{\mathbf{i}} / \mathbf{S}_{\mathbf{i}}^{\mathbf{0}}
$$

3. The evaluation of mutual interest between the coordinator and i-executor

The evaluation of mutual interest between the coordinator and i-executor is determined as minimum of $\mathbf{Z}_{\mathbf{i}}$ and $\mathbf{U}_{\mathbf{i}}$ :

$$
\mathbf{U}_{\mathbf{0 i}}=\min \left(\mathbf{U}_{\mathbf{i}}, \mathbf{Z}_{\mathbf{i}}\right), \mathrm{i}=1,2, \ldots, \mathrm{n}
$$

The received estimations describe separate $\mathrm{CN}$ participants, therefore we name them individual. On the basis of these individual estimations we construct the integrated estimation of $\mathrm{CN}$ participants' interest in cooperation, i.e. we determine the index of structure consolidation (ISC).

\section{The index of structure consolidation (ISC) determination}

To determine the ISC the following procedure is proposed. Let us construct the "petal" diagram. Every axis, starting with the center, is set with the certain executor (and, accordingly, with the certain competency) and scaled from 0 to 1 . This scale shows the relative importance of the particular competency for the project. Then it is necessary to mark 1-points for each axis and connect them. The square of the obtained polygon $\left(\mathrm{F}_{\max }\right)$ shows maximal value of $\mathrm{CN}$ participants' mutual interest in collaboration. It is the "ideal" case, when CN structure is absolutely "compatible". After that we mark $U_{0 i}$ estimations (5) on each axis accordingly and connect the obtained points. The square of the obtained polygon $(\mathrm{F})$ shows real value of $\mathrm{CN}$ participants' mutual interest in collaboration. The index of structure consolidation (ISC) is determined as follows:

$$
\mathrm{ISC}=\mathrm{F} / \mathrm{F}_{\max }
$$

The value of ISC may alter in the interval $[0,1]$. It is necessary to set it to the subjective opinion of coordinator about the "affinity" of CN structure. This procedure claims for fuzzy logic application. The coordinator characterizes the $\mathrm{CN}$ structure "affinity" with several linguistic variables, e.g. weak, medium, and strong. Then the coordinator sets the certain zone of the interval $[0,1]$ with each linguistic variable. In case of three variables (weak, medium, and strong) the interval $[0,1]$ of ISC altering should be divided into three zones by boundary points $\mathrm{q}_{1}$ and $\mathrm{q}_{2}\left(\mathrm{q}_{1}<\mathrm{q}_{2}\right)$. The further actions of coordinator will be different depending on which zone contains the ISC determined previously (6).

If ISC $<\mathrm{q}_{1}$, the level of mutual interest is extremely low, $\mathrm{CN}$ is potentially unstable and the risk of project failure is very high. The coordinator is recommended to change the $\mathrm{CN}$ structure or elaborate additional motivation measures.

If ISC $>q_{2}$, the level of mutual interest is very high, $\mathrm{CN}$ is stable and the risk of project failure is extremely low. The coordinator is recommended to finally select this variant of the $\mathrm{CN}$ structure.

If $\mathrm{q}_{1}<$ ISC $<\mathrm{q}_{2}$, the level of mutual interest is medium, the situation is extremely uncertain. It is necessary to carry out additional analysis, reconsider the structure of accepted requirements, priorities and other elements of the applied selection model.

If necessary, the proposed method is applied also for next modified $\mathrm{CN}$ structure till the coordinator receives the $\mathrm{CN}$ structure that is satisfactory for him. 


\section{ILLUSTRATION}

Let us assume that based on $\mathrm{CN}$ configuration models and algorithms (Ivanov et al., 2004, 2005, 2006, 2007), a CN structure is configured. While analysing CN structure we focuses on the evaluation of its internal "affinity"or "durability". It is necessary to estimate $\mathrm{CN}$ structure stability applying the Index of Structure Consolidation (ISC). Let us assume that the level of structure consolidation can be one of three: low, medium and high. Therefore, boundaries for these three options are set as follows: $\mathrm{q} 1=0,3$ and $\mathrm{q} 2=0,6$. After that we determine the level of mutual interest between the coordinator and each executor $(1,2,3,4,5)$. The result is presented in table 1 .

Table 1 - Level of mutual interest between the coordinator and each executor

\begin{tabular}{|l|c|c|c|c|}
\hline \multicolumn{2}{|c|}{ The level of interest } & \multicolumn{4}{|c|}{ Executors } \\
\cline { 2 - 5 } & $\mathrm{A}$ & $\mathrm{B}$ & $\mathrm{D}$ & $\mathrm{F}$ \\
\hline $\begin{array}{l}\text { The level of coordinator's interest in executor } \\
\left(\mathbf{U}_{\mathbf{i}}\right)\end{array}$ & 0,95 & 0,98 & 0,99 & 0,98 \\
\hline The level of executor's interest in project $\left(\mathbf{Z}_{\mathbf{i}}\right)$ & 0,96 & 0,8 & 0,75 & 0,75 \\
\hline $\begin{array}{l}\text { The level of mutual interest between the coordi- } \\
\text { nator and the executor }\left(\mathbf{U}_{\mathbf{0}}\right)\end{array}$ & 0,95 & 0,8 & 0,75 & 0,75 \\
\hline
\end{tabular}

To determine the ISC it is necessary to construct the "petal" diagram (fig.1) using data presented in table 2 . The level of comparative importance of the project stage (ricom) is determined as follows:

$$
r_{i}^{\text {OTH }}=r_{i} / r_{i}^{\max }
$$

For the stage with maximum level of importance $\left(r_{i}^{\max }\right) r_{i}^{\text {com }}=1$.

The estimation of comparative mutual interest between the coordinator and the executor $\left(\mathrm{U}^{\mathrm{com}}{ }_{0 \mathrm{i}}\right)$ is carried out as follows:

$$
\mathrm{U}^{\mathrm{com}}{ }_{0 \mathrm{i}}=\mathrm{r}_{\mathrm{i}}^{\mathrm{com} *} \mathrm{U}_{0 \mathrm{i}}
$$

Table 2 - Mutual interest between partners

\begin{tabular}{|l|c|c|c|c|}
\hline \multicolumn{1}{|c|}{ Indices } & \multicolumn{4}{c|}{ Executors } \\
\cline { 2 - 5 } & $\mathrm{A}$ & $\mathrm{B}$ & $\mathrm{C}$ & $\mathrm{D}$ \\
\hline The importance of the project stage $\left(\mathbf{r}_{\mathbf{i}}\right)$ & 0,2 & 0,34 & 0,24 & 0,22 \\
\hline $\begin{array}{l}\text { The mutual interest between the coordinator } \\
\text { and the executor }\left(\mathbf{U}_{\mathbf{0}}\right)\end{array}$ & 0,95 & 0,8 & 0,75 & 0,75 \\
\hline $\begin{array}{l}\text { The comparative importance of the project } \\
\text { stage }\left(\mathbf{r}_{\mathbf{i}}^{\text {com }}\right)\end{array}$ & 0,59 & 1 & 0,71 & 0,65 \\
\hline $\begin{array}{l}\text { The comparative mutual interest between the } \\
\text { coordinator and the executor }\left(\mathbf{U}^{\text {com }}{ }_{\text {oi }}\right)\end{array}$ & 0,56 & 0,8 & 0,53 & 0,49 \\
\hline
\end{tabular}




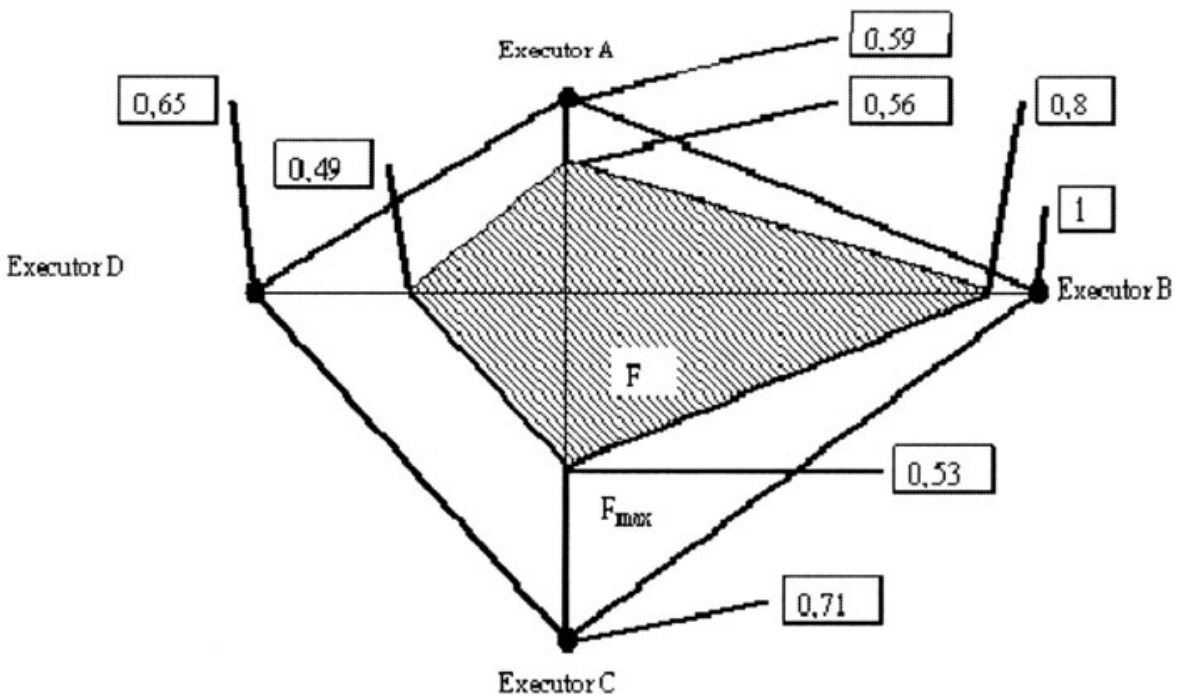

Figure 1. ISC determination

Finally, we obtained the result: $F_{\max }=1,0725, F=0,70305$, ISC $=\mathbf{0 , 6 6}$. It is clear, that ISC $>q_{2}$. It means that the level of mutual interest is very high. From the coordinator's point of view $\mathrm{CN}$ structure is stable enough and the risk of project failure is extremely low. The coordinator is recommended to finally select this variant of the $\mathrm{CN}$ structure. It is reasonable to consider this variant of $\mathrm{CN}$ structure most preferable.

\section{CONCLUSIONS}

Stability analysis is an efficient tool to analyze $\mathrm{CN}$ under the terms of uncertainty. In this paper, we put the emphasis on a specific aspect of the $\mathrm{CN}$ stability analysis analysis of collaboration stability. The elaborated framework aims at developing a technique for simultaneous $\mathrm{CN}$ structuring and its stability assessment based on parametric analysis of possible participants and integral stability assessment. This procedure is based on the application of special index of structure consolidation (ISC), which makes it possible to estimate the project stability. The index shows mutual interest between $\mathrm{CN}$ partners and the coordinator. This index should be taken into account while taking decision about the final structure of the network and its application claims for fuzzy logic introduction. Numerical eexperiments illustrated efficiency of the proposed methods and algorithms. The elaborated framework makes it possible to increase the quality of decision-making about the $\mathrm{CN}$ configuration under the terms of uncertainty.

\section{ACKNOWLEDGMENTS}

The research described in this paper is partially supported by grants from Russian Foundation for Basic Research (RFFI) and German Research Foundation (DFG). The author thanks the contribution from their partners in such projects. 


\section{REFERENCES}

1. Camarinha-Matos, L., Afsarmanesh, H. and A. Ortiz (eds.) (2005). Collaborative Networks and Their Breeding Environments, Springer.

2. Croson, R., H. Donohue, E. Katok and J. Sterman, Order Stability in Supply Chains: Coordination Risk and the Role of Coordination Stock, MIT Press, 2005.

3. Fox, M., A. Gerevini, D. Long and I. Serina, Plan Stability: Replanning versus Plan Repair, in: Proceedings of the ICAPS'2006, 2006.

4. Heisig, G. Planning Stability in Material Requirements Planning Systems, Gabler Verlag, 2006.

5. Ivanov, D., A. Arkhipov, V. Tolkacheva and B. Sokolov, Stability Analysis in the Framework of Decision Making Under Risk and Uncertainty, in: Network-centric collaboration and supporting frameworks, L.M. Camarinha-Matos, H. Afsarmanesh, M. Ollus (eds.), Springer, 2006, pp. 211 218.

6. Ivanov, D., Arkhipov A., Sokolov B. (2004): Intelligent Supply Chain Planning in Virtual Enterprises. In: Virtual Enterprises and Collaborative Networks, edited by L.Camarihna-Matos, Kluwer Academic Publishers, 2004: 215-223.

7. Ivanov, D. DIMA - Decentralized Integrated Modeling Approach. Interdisziplinäre Modellierung von Produktions- und Logistiknetzwerken (2007), Verlag der GUC, Chemnitz, 2007

8. Ivanov, D., B. Sokolov (2007) Intelligent planning and control of manufacturing supply chains in virtual enterprises, accepted IJMTM, 2007.

9. Ivanov, D., Käschel, J., Arkhipov, A., Sokolov, B., and Zschorn L. (2005): Quantitative Models of Collaborative Networks, In: Collaborative Networks and Their Breeding Environments, edited by L.Camarihna-Matos, H. Afsarmanesh, A. Ortiz, Springer, 2005, pp. 387-394.

10. Kulba, V., B. Pavlov, and O. Zaikin, Stuctural-technological reserve for increasing stability of manufacturing systems', in: Proceedings of the 12th IFAC Symposium on Information Control Problems in Manufacturing, St. Etienne, France, 2006, Vol. 2, pp. 93-98.

11. Rouche, N., P. Habets and M. Laloy, Stability Theory by Liapunov's Direct Method, Springer, 1977. 